\title{
ANÁLISE DAS DIMENSÕES DE FLEXIBILIDADE DE MANUFATURA PARA A PREVENÇÃO DE PROBLEMAS EM UMA INDÚSTRIA DE PNEUS
}

\author{
ANÁLISIS DE LAS DIMENSIONES DE FLEXIBILIDAD DE FABRICACIÓN PARA LA \\ PREVENCIÓN DE PROBLEMAS EN UNA INDUSTRIA DE NEUMÁTICOS
}

\section{PROBLEM MITIGATION THROUGH MANUFACTURING FLEXIBILITY DIMENSIONS: ANALYSIS IN A TIRE INDUSTRY}

\author{
Ualison Rebula de Oliveira * \\ Ualison.oliveira@gmail.com \\ Fernando Augusto Silva Martins ** \\ Fernando.marins@outlook.com \\ Henrique Martins Rocha *** \\ Fernando.marins@outlook.com \\ * Universidade Federal Fluminense, São Paulo - Brasil \\ ** Universidade Estadual Paulista. Rio de Janeiro - Brasil \\ *** Universidade do Estado do Rio de Janeiro. Rio de Janeiro - Brasil
}

\section{Resumo Resumen Abstract}

O ambiente onde se desenvolvem as operações de manufatura se constitui de um natural espaço gerador de riscos, sujeito à ocorrência de problemas de diversas naturezas. Como solução, ou minimização dos efeitos indesejados desses problemas, diversos autores recomendam a adoção da Flexibilidade de Manufatura. Entretanto, sua característica multidimensional e, muitas vezes, as particularidades a cada empresa, dificultam a correta associação dos tipos de flexibilidade aos problemas existentes. Metodologicamente, a pesquisa foi suportada por um estudo de campo, baseado em levantamento da percepção dos funcionários de nível gerencial do departamento de produção de uma indústria de pneus, foi analisado os principais problemas que afetam seu ambiente operacional e os tipos de flexibilidades mais requeridos por essa empresa para a solução de seus problemas. Como resultados empíricos, identificou-se que o problema mais crítico está relacionado com equipamentos e que apenas três tipos de flexibilidade de manufatura são demandados pela empresa estudada.

PalaVras ChaVE: Flexibilidade de manufatura; Problemas; Prevenção; Pneus.

El entorno donde se desarrollan las operaciones de fabricación es un espacio natural generador de riesgos, sujeto a la aparición de problemas de diversos tipos. Como solución, o para minimizar los efectos no deseados de estos problemas, varios autores recomiendan la adopción de Flexibilidad de fabricación. Sin embargo, sus características multidimensionales $y$, muchas veces, las particularidades de cada empresa hacen que sea difícil asociar correctamente los tipos de flexibilidad con los problemas existentes. Metodológicamente, la investigación fue apoyada por un estudio de campo, basado en una encuesta de las percepciones de nivel gerencial del departamento de producción de una industria de neumáticos, analizando los principales problemas que afectan su entorno operativo y los tipos de flexibilidades más requeridos por esta empresa para resolver sus problemas. Como resultados empíricos, se identificó que el problema más crítico está relacionado con el equipo y que la compañía estudiada solo exige tres tipos de flexibilidad de fabricación. 
PALABRAS CLAVE: Flexibilidad de fabricación; Problemas; Prevención; Neumáticos.

Manufacturing operations are developed in risk-generating environment, subject to the occurrence of problems of several types and sources. As solution, or at least as problem effect mitigation action, several authors recommend adopting the Manufacturing Flexibility. However, its multidimensional characteristics and, often, each company needs uniqueness hamper the correct selection of the types of flexibility to the existing problems. Even worse, the study of manufacturing flexibility in Brazil is scarce. Methodologically, the research was supported by a field study. The present study describes an empirical research in a tire industry, based on main problems and types of flexibility perception from production management level employees. The survey prospected their perception about problems affecting operation environment and types of flexibilities required to solve/mitigate those problems. As a result, it is observed that the main problem production equipment-related. Also, it has been noticed that, despite the vast typology of manufacturing flexibilities, only three are identified at the company.

KEYWORDS: Manufacturing flexibility; Problems; Prevention; Tires.

\section{Introdução}

Nas últimas décadas presenciou-se uma série de transformações da economia mundial, com nítidos impactos sobre o comércio mundial, a relação entre as nações e a vida das empresas. Tais transformações e inovações desencadearam uma profunda reestruturação em todas as atividades industriais e de serviços, bem como nas estruturas das organizações e no próprio comportamento das pessoas. Conforme Patel et al. (2012), a incerteza do ambiente afeta diretamente o desempenho das organizações. Nesse ambiente, a sobrevivência da empresa depende não apenas de buscar a eficiência nos custos e a liderança na qualidade, mas, também, de estar preparada para incertezas, riscos e instabilidades (OLIVEIRA, 2009).

No passado, mudanças aconteciam lentamente, havendo tempo para que as organizações adaptassem seus processos às oportunidades apresentadas pelo ambiente competitivo. Porém, com a velocidade das mudanças nos dias atuais, as organizações necessitam atuar proativamente nos processos evolutivos e adaptativos, para garantir uma combinação eficiente entre a organização e o ambiente de negócios (DE OLIVEIRA et al., 2017).

Nesse contexto, gerenciar adequadamente os riscos e as condições de incertezas a que a empresa é exposta, significa possibilitar que a organização tenha futuro (BARALDI, 2005), ideia corroborada por Padovese e Bertolucci (2005), que afirmam que a exposição ao risco é um dos maiores desafios à sobrevivência das organizações.

Várias pesquisas sobre o tema gerenciamento de riscos no ambiente produtivo industrial, entre elas as de Slack (1993), Swamidass e Newell (1987), Beach et al. (2000), Kais e Kara (2005), Gerwin (2005), Patel (2011), Purwanto et al. (2015) e outros que compõem o referencial teórico da presente pesquisa, comprovam que vários problemas que ocorrem na manufatura podem ser solucionados, preventivamente, por diferentes tipos de flexibilidade de manufatura. Até mesmo aspectos relacionados à inovação de produtos podem ser influenciados pela flexibilidade de manufatura (OKE, 2013). 
A característica multidimensional da flexibilidade dificulta a tarefa de se estabelecer o grau de adoção, frente às variáveis existentes. De acordo com Upton $(1994,1995)$ e Boyle (2006), a flexibilidade em manufatura pode existir em diferentes níveis da organização e é composta por vários tipos, como flexibilidade de máquinas, de roteirização e de produto. Também a flexibilidade pode ser caracterizada como potencial, real e necessária, conforme a quantidade de estados que podem ser alcançados (gama), da mobilidade (impactos gerados por mudanças, tais como custos e tempo para setup) e da uniformidade (como medidas de desempenho se alteram com as mudanças executadas).

Nessa complexa área do conhecimento, defendida por Slack (1993) como uma das cinco prioridades competitivas em gestão de operações, surge a necessidade de pesquisas que suportem a seleção de flexibilidades de manufatura adequadas à prevenção de diferentes tipos de problemas, visto que a escolha indevida de tipos de flexibilidade de manufatura impactará em investimentos desnecessários ou inadequados, gerando perda de capital e a indisponibilidade e ineficiência dos recursos flexíveis escolhidos.

Diante dessa contextualização, julga-se cabível a tentativa de se desenvolver uma abordagem diferenciada para a prevenção de riscos na manufatura, com base nos conceitos de flexibilidade de manufatura, que ajude as organizações a minimizarem os problemas que ocorrem em seus processos produtivos. $O$ presente trabalho buscou identificar os principais problemas que ocorrem na manufatura de uma indústria de pneus e quais tipos de flexibilidades são indicados para compor um grupo ideal de flexibilidades para resolver esses problemas. Como resultados, a empresa analisada possuirá um estudo que lhe permita minimizar recursos destinados ao investimento para se tornar flexível.

Quanto à estrutura, o artigo se subdivide em outras quatro seções (metodologia, referencial teórico, resultados da pesquisa e conclusões), conforme segue.

\section{Método}

Para a presente pesquisa, adotou-se o método hipotético-dedutivo, conforme a Figura 1.

Figura 1: Esquema do método para a pesquisa 
Conhecimento prévio e teorias existentes:

Flexibilidade de manufatura; Gerenciamento de riscos; Riscos e incertezas na Manufatura.

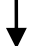

Lacuna, contradição ou problema:

Quais são os tipos de flexibilidade de manufatura que, isolados ou aglutinados, melhor previnem os

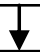

\section{Conjecturas:}

$\mathbf{C}_{1}$. Os problemas críticos na manufatura estão relacionados aos fornecedores e com a indisponibilidade de equipamentos.

$\mathbf{C}_{2}$. Os problemas mais frequentes na manufatura estão ligados aos atrasos de entrega de matéria-prima por fornecedores e à falhas de comunicação.

$C_{3}$. As flexibilidades de máquina e de mão-de-obra são os tipos que mais ajudam na solução de problemas da indústria.

\section{Consequências falseáveis, enunciados deduzidos}

Se as conjecturas formuladas não forem verdadeiras, será possível concluir que as possíveis soluções não

\section{Etapa de Verificação}

Questionários direcionados a tipos específicos de respondentes (pessoas que trabalham na manufatura, em funções operacionais, de chefia, supervisão e gerência). Realização de pré-testes para avaliação da clareza e o tempo necessário para o preenchimento dos instrumentos formulados. Reformulação do questionário até

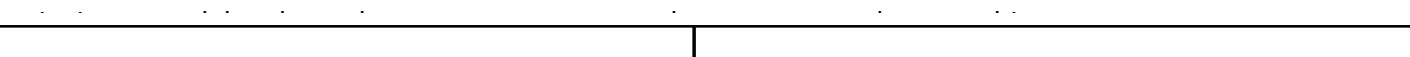

Análise dos resultados

Os dados colhidos na pesquisa de campo serão analisados por meio de método estatístico.

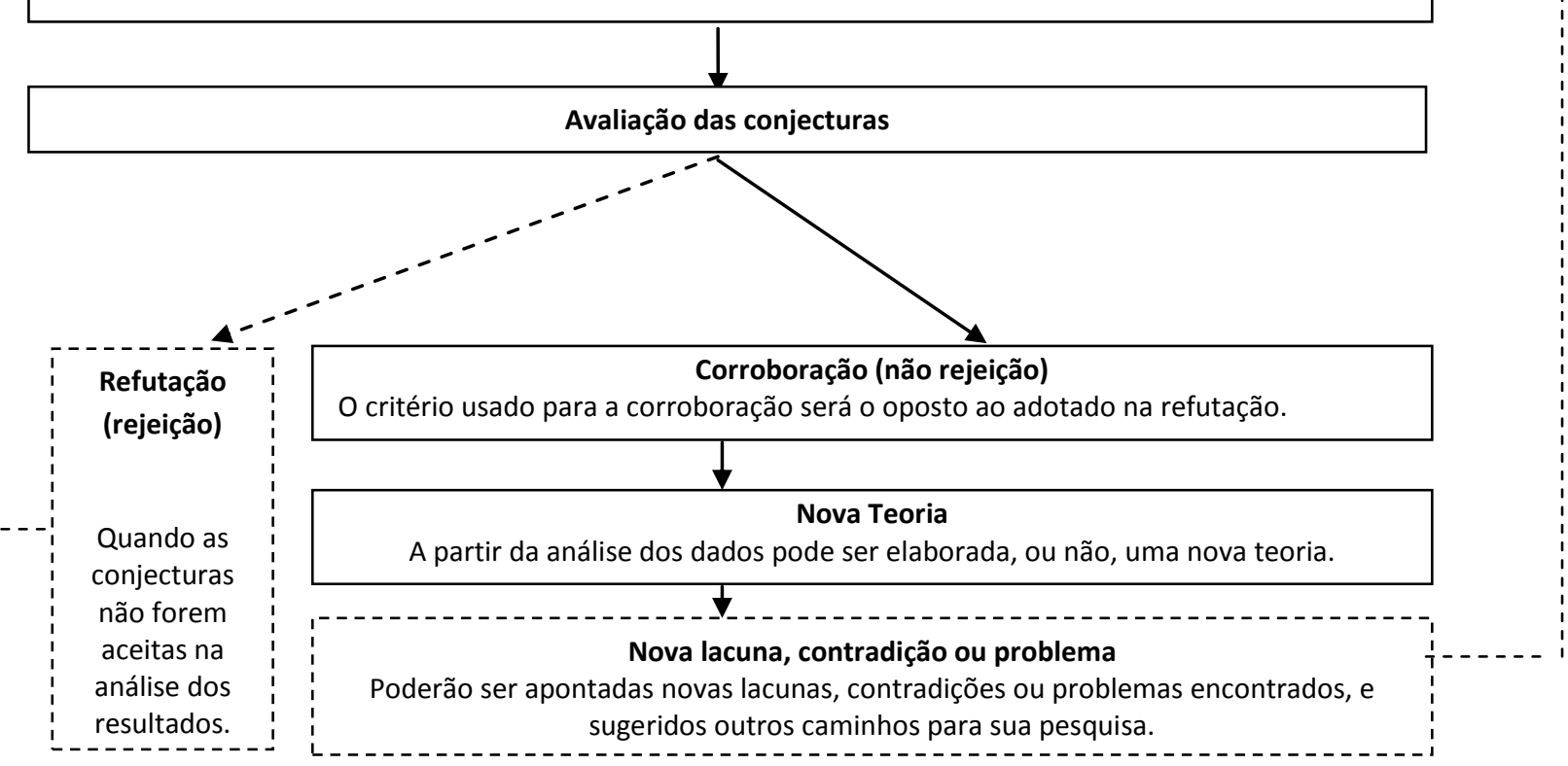

Fonte: Adaptado de Marconi e Lakatos (2004) 
Utilizou-se o método de abordagem hipotético-dedutivo que parte da percepção de uma lacuna nos conhecimentos, acerca da qual se formulam conjecturas. Pelo processo de inferência dedutiva, testa-se a predição da ocorrência de fenômenos abrangidos pelas conjecturas (MARCONI; LAKATOS, 2004). Quanto aos métodos e procedimentos adotados, Marconi \& Lakatos (2006) defendem que o estudo de determinados indivíduos, profissões e instituições (método monográfico) permite comparações com a finalidade de verificar similaridades e explicar divergências (método comparativo), reduzindo os fenômenos sociais, políticos, econômicos, etc. a termos quantitativos, enquanto a manipulação estatística permite comprovar as relações dos fenômenos entre si e obter generalizações sobre a natureza, ocorrência ou significado (método estatístico).

Como há várias taxonomias de flexibilidade de manufatura, muitas vezes conflitantes e sobrepostas (D'SOUZA; WILLIAMS, 2000; UPTON, 1997; SAWHNEY, 2005), selecionaram-se apenas as mais relevantes (SLACK, 1983; GUPTA; GOYAL, 1989; KOSTE; MALHOTRA, 1999; VOKURKA; O'LEARY-KELLY, 2000) para o desenvolvimento do instrumento de coleta de dados, quais sejam: Flexibilidade de entrega; Flexibilidade de expansão; Flexibilidade de máquina; Flexibilidade de mão-de-obra; Flexibilidade de mix; Flexibilidade de modificação; Flexibilidade de movimentação de material; Flexibilidade de operação; Flexibilidade de produção; Flexibilidade de produto; Flexibilidade de roteamento; e Flexibilidade de volume.

Sem o intuito de generalizar resultados para outras organizações no mesmo segmento, esse artigo focou a influência das dimensões da flexibilidade sobre os riscos e problemas que ocorrem na manufatura, com base na percepção dos funcionários no ambiente produtivo de uma grande indústria de pneus localizada no estado do Rio de Janeiro. O estudo utilizou uma amostra de cinco respondentes de nível tático/gerencial, com o objetivo principal de pesquisar as dimensões de flexibilidade de manufatura requeridas pela referida empresa, como forma de se antecipar aos problemas que ocorrem em seu processo produtivo.

A seleção da empresa se deu por conveniência (o pesquisador possuía facilidade de acesso para realizar a pesquisa). O critério utilizado para seleção dos respondentes foi do tipo não probabilístico intencional, onde a seleção dos respondentes se deu por julgamento (o pesquisador escolheu os elementos que julgou serem mais representativos da população): Os respondentes possuíam cargos de chefia e trabalhavam no processo produtivo industrial da empresa pesquisada. Todos possuíam, no mínimo, curso de graduação e eram coordenadores, supervisores ou gerentes de produção. A pequena, porém representativa, amostra foi composta por indivíduos que atuavam diretamente na gestão do ambiente de operações de produção e compunham o staff decisor de manufatura.

Adotou-se o questionário como instrumento de coleta de dados, no qual se buscava identificar a percepção dos respondentes sobre os tipos de problemas que mais impactavam as operações, sua escala de importância, abrangência e frequência e quais tipos de flexibilidade poderiam ser utilizados para solucioná-los. Optou-se pelo questionário, composto de perguntas abertas e fechadas, com escalas atitudinais e com classificação de prioridades tipo likert, como forma rápida e de baixo custo para coletar as informações necessárias à pesquisa, com facilidade de preenchimento, buscando minimizar os riscos de vieses nos resultados. 
As questões do questionário foram elaboradas de forma a refutar ou aceitar as conjecturas que constam na Figura 1, cujos resultados são detalhados no capítulo 4.

Antes da submissão dos questionários, testes pilotos foram aplicados, visando identificar o que ocorreria numa situação real de coleta de dados, procedendo com as revisões necessárias para minimizar interpretações equivocadas, preenchimentos incorretos e outros inconvenientes que podem ocorrer nesse tipo de pesquisa. Ressalta-se que as pessoas que participaram do teste piloto não fizeram parte da amostra dos cinco respondentes selecionados.

\section{Referencial teórico sobre flexibilidade de manufatura}

De acordo com Narasimhan et al. (2004), a literatura sobre flexibilidade de manufatura contém quatro temas distintos, porém relacionados:

Fatores contextuais, em que a necessidade de fazer face à incerteza ambiental de volume e variedade é o principal motivo para desenvolver a flexibilidade em manufatura;

Classificações e taxonomias de flexibilidade de manufatura, enfatizando a natureza multidimensional do constructo;

Antecedentes da flexibilidade, identificando as principais influências estruturais e de infraestruturas, tais como tecnologia, mão-de-obra, gestão da cadeia de suprimentos, projeto do trabalho e controle de produção;

- $\quad$ Relação entre a flexibilidade de manufatura e certos aspectos do desempenho de manufatura.

A partir dessa observação, os aspectos mais relevantes correlacionados à pesquisa são abordados aqui, permitindo uma melhor compreensão do objeto de estudo.

\section{III.I. Histórico e conceito de flexibilidade de manufatura}

Suarez et al. (1995) defendem que a flexibilidade coloca-se com tanta importância estratégica como foram o gerenciamento científico no início do século XX e a qualidade que emergiu em meados do mesmo século; o que também é defendido por De Toni \& Tonchia (1998).

Historicamente, observa-se que a flexibilidade de manufatura começou a ser pesquisada, para administrar operações sob condições de variedade e incertezas (HE et al., 2014), a partir da década de 30, quando Stigler (1939 apud UPTON, 1995, p. 207) desenvolveu pesquisas que relacionavam a flexibilidade de volume com pequenos lotes de produção. $\mathrm{Na}$ década de 60, Nelson (1967 apud KOSTE; MALHOTRA, 1999, p. 80) ampliou a atuação da flexibilidade de manufatura, relacionando-a com sistemas limitados de produção e Skinner (1969 apud BOYLE 2006, p. 7) identificou flexibilidade como um dos quatro objetivos da manufatura, ao lado dos custos de produção, entrega e qualidade. Na década de 70, Fryer (1974) relacionou a flexibilidade com a mão-de-obra. 
A partir da década de 80, o assunto despertou o interesse de vários outros pesquisadores, dos quais se destacam: Slack $(1983 ; 2005)$ - flexibilidade como um objetivo a ser atingido na manufatura; Gustavsson (1984) - flexibilidade e produtividade em sistemas produtivos complexos; Browne et al. (1984) - flexibilidade para planejamento do futuro incerto; Swamidass \& Newell (1987) - flexibilidade em ambientes de incerteza; Gerwin (1987) Flexibilidade em processos de manufatura; e Gupta e Goyal (1989) - Dimensões da flexibilidade de manufatura.

De acordo com Vokurka \& O'Leary-Kelly (2000) e De Treville et al. (2007), desde que Hayes e Wheelwright defenderam em 1984 a idéia de que a flexibilidade em manufatura era uma das principais dimensões de estratégia competitiva de negócios, houve um crescimento substancial na quantidade de pesquisas sobre o tema. Entretanto, apesar de sua relevância, no Brasil, os estudos sobre flexibilidade de manufatura ainda são escassos (OLIVEIRA, 2009).

Sua adoção pelas organizações, independentemente dessas serem de grande ou pequeno porte (MISHA, 2016), promove melhor capacidade de reação diante dos riscos e incertezas.

Quanto ao seu significado, Vokurka e O'Leary-Kelly (2000) definem flexibilidade em manufatura como sendo a habilidade das empresas em reagir às mudanças nas necessidades dos clientes, bem como às mudanças imprevistas decorrentes de pressões concorrenciais. Segundo os autores, trata-se de uma ferramenta chave para a competitividade, com crescente variedade de produtos, os quais têm ciclos de vida cada vez mais curtos; conceito este também defendido por Pagell e Krause (1999; 2004) e Oke (2005). Isso exige esforço e criatividade das empresas e, conforme Bolwijn e Kumpe (1990), não é possível ser inovador sem ser flexível.

Watts et al. (1993) definem flexibilidade como a habilidade de implementar mudanças no ambiente operacional interno, em tempo adequado e custo aceitável, em resposta às mudanças nas condições de mercado. Já para Olhager (1993), seria a habilidade de se adaptar às mudanças de condições utilizando os recursos existentes (em curto prazo) e de introduzir novos produtos, recursos e métodos de produção, incorporando-os ao sistema de produção existente.

\section{III.II. Motivos pelo quais as empresas demandam flexibilidade de manufatura}

Slack (2005) cita que com a crescente demanda dos consumidores por rápidas respostas e grande variedade de produtos, aliado a um cenário de competição cada vez mais acirrada, flexibilidade é a solução para muitos problemas do gênero. Segundo Bengtsson e Olhager (2002), para reagir ao aumento das mudanças, da redução do ciclo de vida dos produtos e da comercialização globalizada, a flexibilidade está se tornando uma importante fonte de vantagem competitiva para a manufatura. Nesta mesma linha, Serrão (2001) defende que a flexibilidade de manufatura se tornou um elemento de diferenciação da competitividade entre as empresas.

Slack (1993) classifica a flexibilidade como uma fonte de vantagem competitiva, advertindo que a manufatura necessita ser flexível para lidar com a operação sob condições de 
variedade, riscos e incertezas, permitindo que o processo produtivo prossiga a contento. Conforme o autor, a flexibilidade contribui para o desempenho da manufatura, melhorando variáveis como a confiabilidade, custos e velocidade. Quase 25 anos após a pesquisa de Slack (1993), Choe et al. (2015) continuam defendendo a flexibilidade de manufatura como uma estratégia competitiva frente às incertezas do mercado.

Com a flexibilidade, as empresas são capazes de produzir produtos com qualidade superior orientados para o cliente, a um custo baixo e com uma resposta mais rápida às alterações do mercado (WAHAB et al., 2008). Segundo Kara e Kayis (2004), a conveniência de flexibilidade em manufatura decorre da capacidade das organizações lidarem eficazmente com a incerteza oriunda de diversas fontes, opinião compartilhada por Swamidass e Newell (1987) e Gupta e Somers (1992).

Correa (1994) julga que a flexibilidade surge por conta da necessidade de lidar com incertezas intrínsecas e variabilidade de saídas de processos. As incertezas e variabilidades levam às mudanças, sendo estas tanto planejadas (acontecem como decisão consciente da organização, tomada para alterar a própria organização ou sua relação com o ambiente) como não planejadas (acontecem independentemente da determinação da organização, mas as quais a organização precisa se adaptar). Por exemplo, quando o tempo requerido pelo cliente é inferior ao tempo de produção, ou quando o cliente muda a quantidade ou prazo dos pedidos, a flexibilidade aumentará a capacidade de atender às necessidades deste cliente (KARA e KAYIS, 2004). Várias outras abordagens sobre a capacidade de flexibilidade de manufatura atuar frente aos riscos e incertezas podem ser consultadas na literatura sobre esse tema. Urtasun-alonso et al. (2014), por exemplo, relacionam práticas avançadas de Gestão de Pessoas com a Flexibilidade de Manufatura. Fernandes et al. (2012) abordam o uso de opções reais para investimentos em equipamentos como resposta rápida de demandas incertas.

Correa (1994) sugere que incerteza ambiental e variabilidade nos resultados são as duas principais razões (ou estímulos) para a busca da flexibilidade de manufatura, as quais podem ser traduzidas em alterações operacionais previstas e/ou não planejadas. As fontes de estímulos podem ser processo, fornecedores, clientes, sociedade, Corporação, outras funções e concorrentes. Segundo o autor, as mudanças não planejadas têm cinco dimensões principais: tamanho, novidade, frequência, certeza e taxa. Como consequência, os gestores buscam manter controle sobre a situação, sendo a flexibilidade a forma buscada, como ilustra a da Figura 2.

Figura 2: Ligações entre dimensões de mudança e flexibilidade 


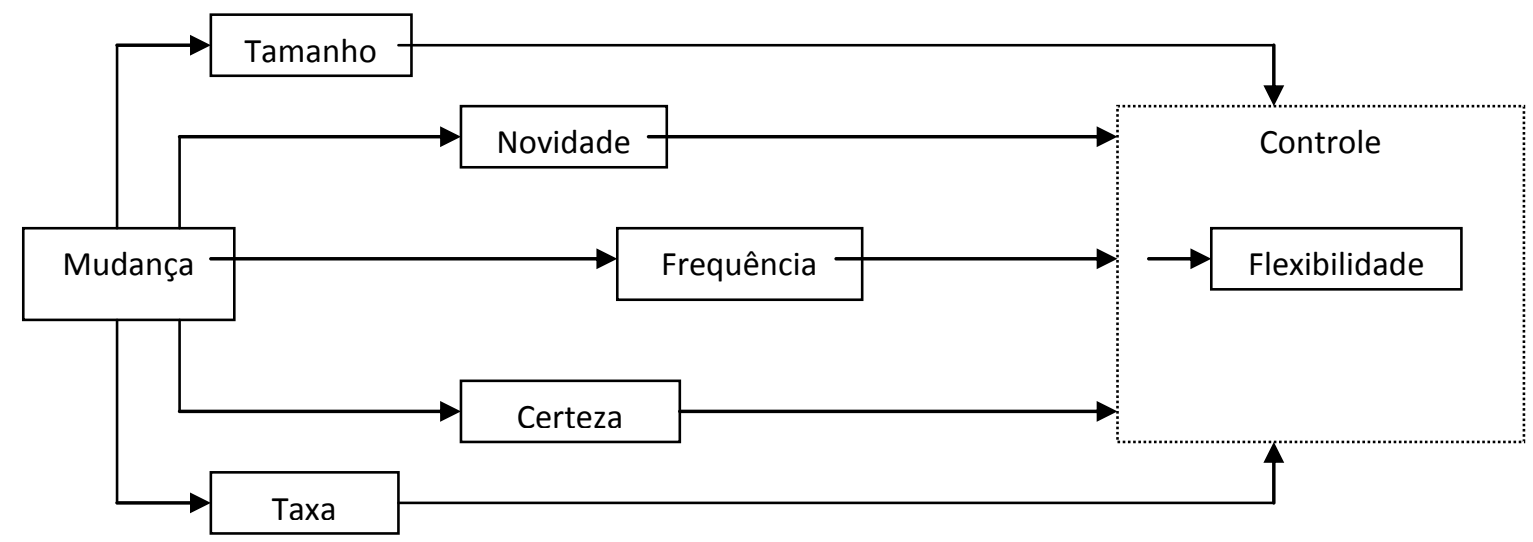

Fonte: Correa (1994)

A flexibilidade de manufatura é necessária para prover as organizações de condições de alterar os níveis de produção rapidamente, para desenvolver novos produtos mais rapidamente e com maior frequência e para responder rapidamente às ameaças competitivas (OKE, 2005; CHANG et al., 2007; JAVIER et al., 2014). Kara e Kayis (2004) descrevem as necessidades que levam à flexibilidade, as quais são apresentadas na Figura 3.

Figura 3: Fatores causais da necessidade de flexibilidade

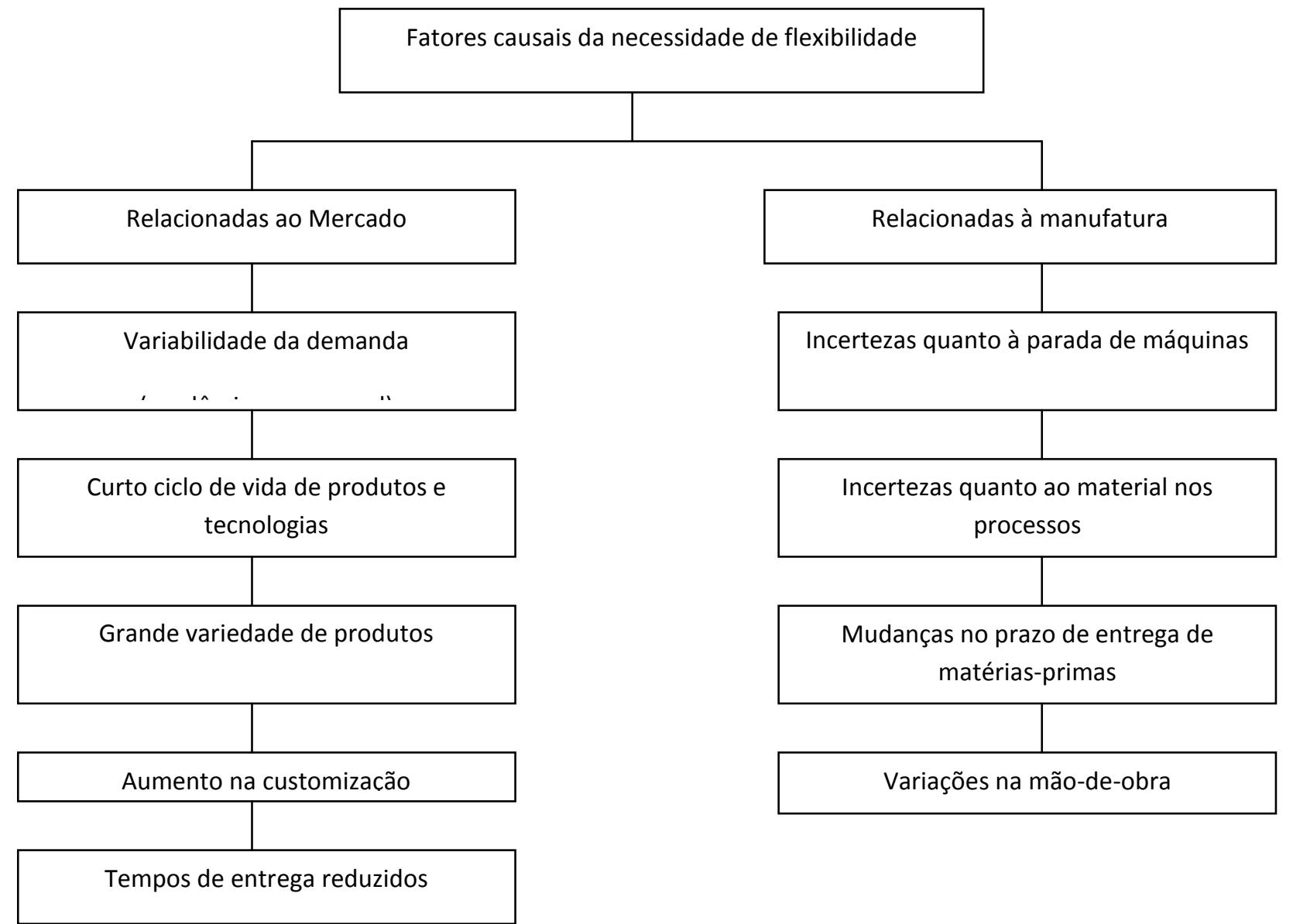

Fonte: Kara e Kayis (2004) 


\section{III.III. Motivos pelo quais as empresas demandam flexibilidade de manufatura}

Como resultado de sua natureza multidimensional, a flexibilidade não é genérica e deve ser cuidadosamente justificada, planejada e gerida, de forma a ser bem sucedida na sua implementação e gerar os resultados esperados (BEACH et al., 2000). Sua implementação não demanda, necessariamente, requisitos tecnológicos (MISHRA et al., 2016).

Para atingir flexibilidade em manufatura, Slack (1983), recomenda uma abordagem em três fases:

1) Definir requisitos de flexibilidade, entendendo a estratégia da organização;

2) Realizar auditoria de flexibilidade, para determinar a capacidade do sistema atual em termos de gama e velocidade; e

3) Desenvolver programa de ação para reduzir a lacuna existente entre a flexibilidade necessária e as capacidades atuais.

Olhager e West (2002), propuseram uma "Casa de Flexibilidade" (Figura 4), cuja abordagem é baseada na Casa da Qualidade, desenvolvida para auxiliar as empresas a desdobrar as demandas de mercado quanto à flexibilidade em competências para a flexibilidade, identificando os impactos e oportunidades existentes.

Figura 4: Relacionando habilidades e características de flexibilidade

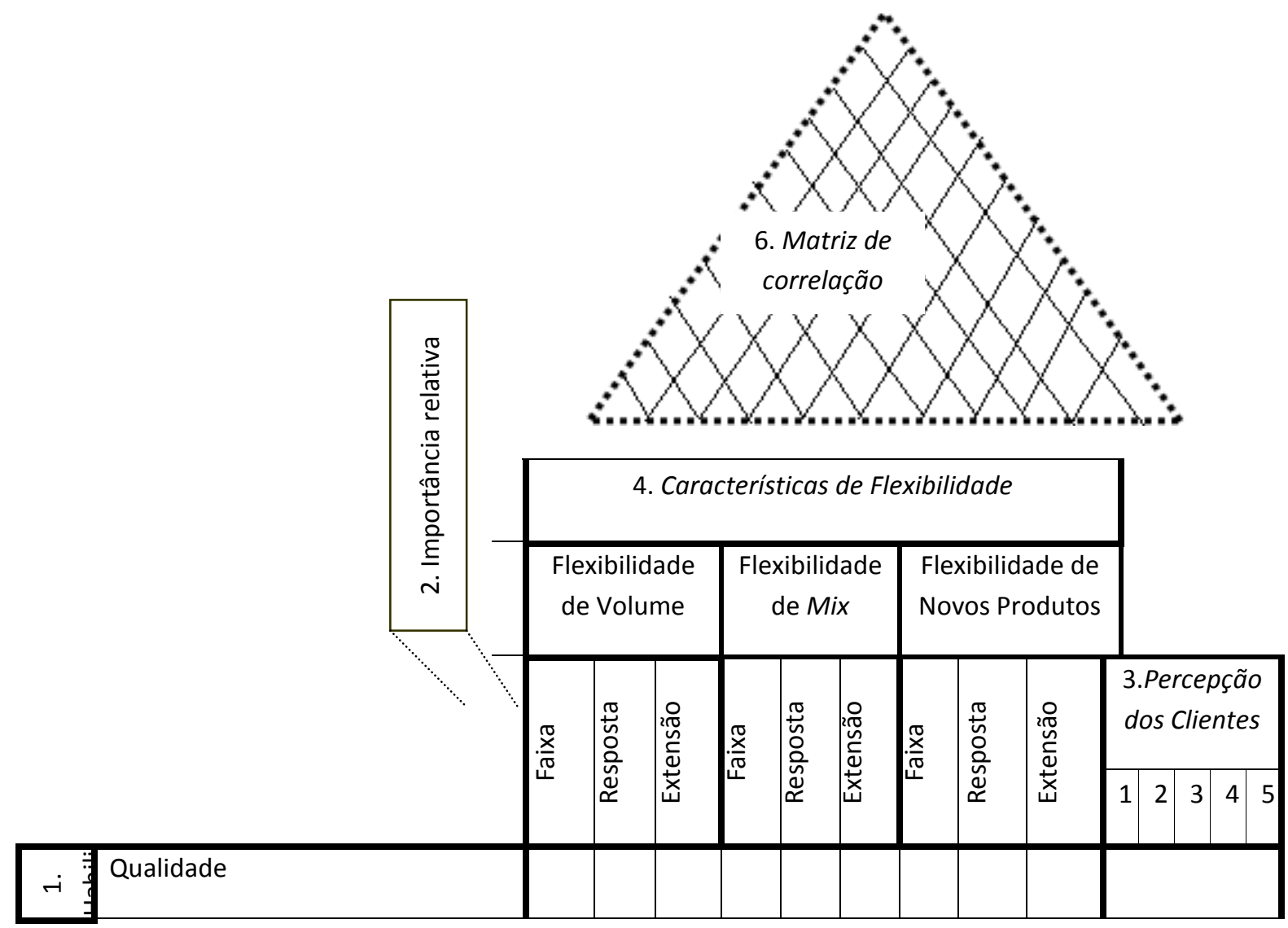




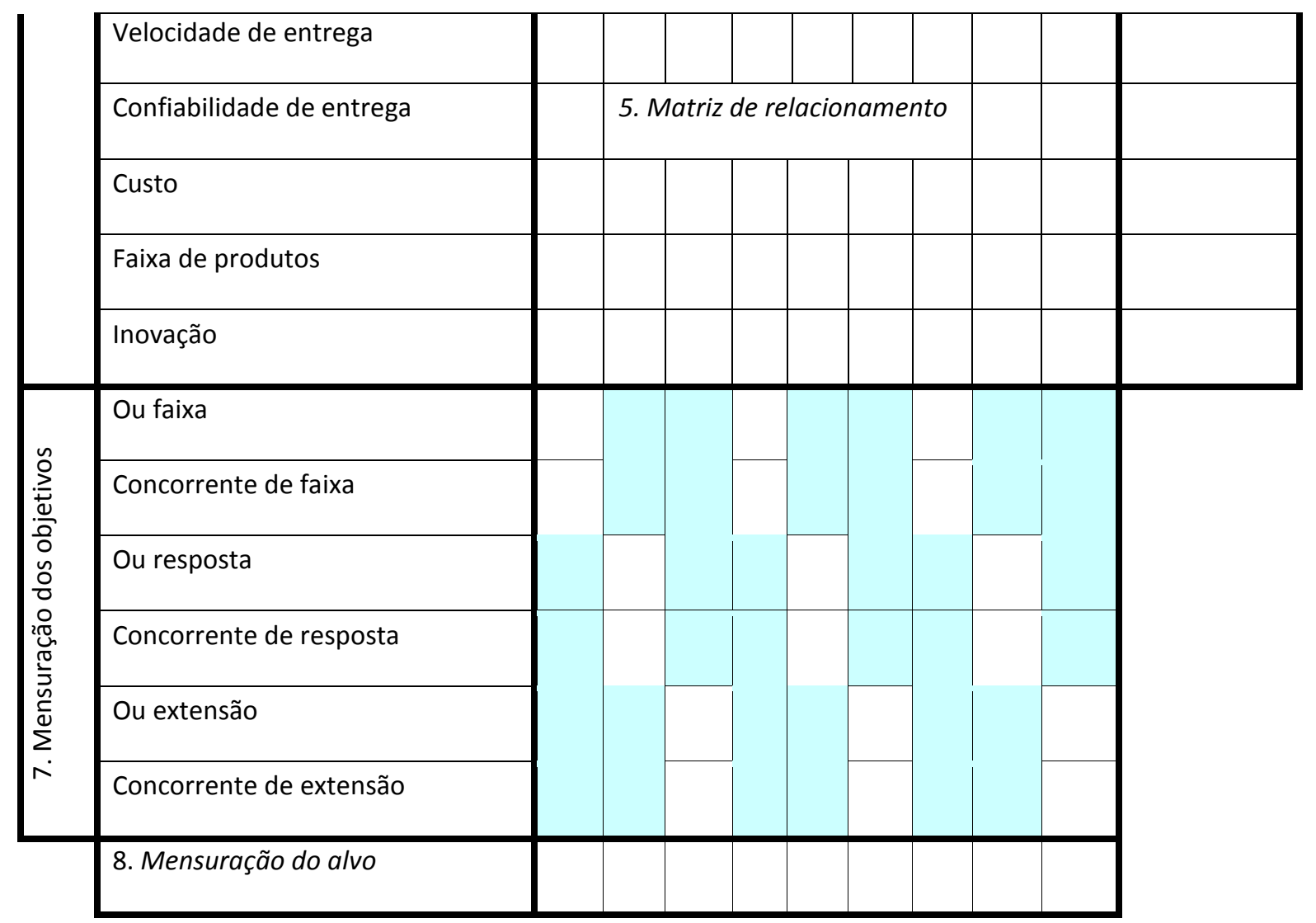

Fonte: Olhager e West (2002

Boyle (2006) avaliou os modelos propostos por Olhager e West (2002), Narain et al. (2000), Nilsson \& Nordahl (1995) e Gerwin (1993), coletando nestes estudos uma lista de melhores práticas para a implementação da flexibilidade em manufatura, propondo o modelo que pode ser observado na Figura 5.

Figura 5: Modelo para implementação de flexibilidade em manufatura 


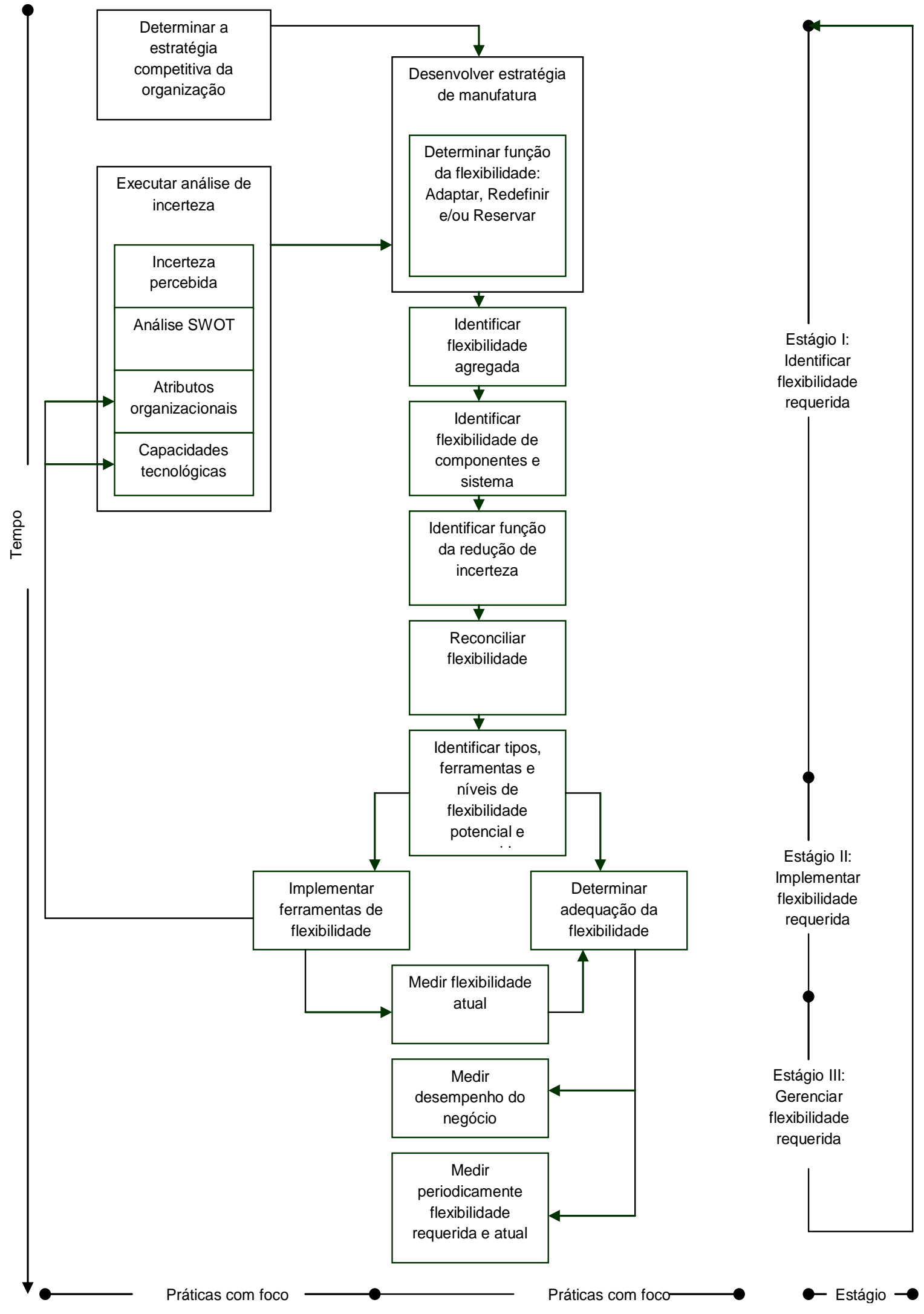

Fonte: Boyle (2006) 


\section{Pesquisa empírica em uma indústria de pneus}

A pesquisa teve sua primeira fase voltada à análise dos problemas que ocorrem no processo de manufatura de uma indústria de pneus, enquanto a segunda identificou as dimensões de flexibilidades requeridas por essa empresa.

\section{IV.I. Análise dos problemas que ocorrem na manufatura}

Coletadas as respostas aos questionários, observou-se que os problemas mais críticos na indústria de pneus estavam relacionados, nesta ordem, à indisponibilidade de equipamentos, falha humana, fornecedores (aqui se inclui, também, concessionárias de energia elétrica), erros de planejamento, falhas de comunicação, retrabalho e problemas de natureza ambiental. Também se constatou que, geralmente, os problemas mais críticos não eram os mais frequentes. Por exemplo, as falhas de comunicação, eram mais frequentes, porém, menos críticas.

Outras informações sobre os problemas que ocorriam na manufatura da empresa pesquisada, podem ser visualizadas no Quadro 1, desenvolvido para condensar e facilitar a interpretação dos dados. A seguir, a descrição dos campos do mesmo:

A coluna 'tipo de problema' informa os tipos de problemas que ocorrem na empresa pesquisada, segundo a percepção do respondente, aglutinando-os por similaridade; os números entre colchetes representam quantos respondentes citaram o mesmo problema;

A coluna 'vezes citada' informa o total de vezes que problemas de uma mesma família (problemas similares) foram citados pelo respondente;

A coluna 'classificação' ordena os problemas por grau de impacto negativo à organização pesquisada, de forma ordinal; quanto menor o número, pior é o problema para a organização;

A coluna 'média p' informa a média das classificações ordinais da coluna anterior;

A coluna 'frequência' informa com qual frequência os problemas citados na primeira coluna (tipo de problema) ocorrem na organização pesquisada; os números entre parênteses representam o valor cardinal das frequências: nunca (5), raramente (4), às vezes (3), frequentemente (2) e sempre (1);

Por fim, a última coluna 'flexibilidade escolhida para solucionar o problema' pode conter uma quantidade de flexibilidades superiores à quantidade de problemas apontados na primeira coluna. Isso ocorre porque foi permitido ao respondente recomendar quantos tipos de flexibilidade que ele julgasse necessário para mitigar ou até mesmo resolver os problemas apontados.

\section{IV.I. Análise de flexibilidade de manufatura}


Ainda analisando o Quadro 1, especificamente a coluna "flexibilidade escolhida para solucionar o problema", observa-se que, para a solução dos problemas apontados na subseção anterior, as flexibilidades mais recomendadas na indústria de pneus são as flexibilidade de máquina, de mão de obra e de volume, sendo esta última indicada para erros de planejamento de vendas.

Quadro 1: Análise de incertezas, riscos e problemas para a indústria de pneus

\begin{tabular}{|c|c|c|c|c|c|c|c|}
\hline \multicolumn{8}{|c|}{ INDÚSTRIA DE PNEUS - ANÁLISE DE INCERTEZAS, RISCOS E PROBLEMAS } \\
\hline Tipo de problema & $\begin{array}{l}\text { Vezes } \\
\text { citada }\end{array}$ & \begin{tabular}{|c} 
Classificaçã \\
o
\end{tabular} & $\begin{array}{l}\text { Média } \\
\text { P }\end{array}$ & Frequência & $\begin{array}{c}\text { Média } \\
\text { F }\end{array}$ & \begin{tabular}{|c|} 
Fato \\
$r$ \\
PF/V
\end{tabular} & $\begin{array}{c}\text { Flexibilidade } \\
\text { escolhida para } \\
\text { solucionar o } \\
\text { problema }\end{array}$ \\
\hline $\begin{array}{l}\text { Quebra de } \\
\text { máquina [3]; mau } \\
\text { funcionamento de } \\
\text { equipamentos [1]; } \\
\text { máquina não } \\
\text { funcionar por } \\
\text { poeira [1]. }\end{array}$ & 5 & $1^{\circ} ; 1^{\circ} ; 2^{\circ} ; 2^{\circ} ; 1^{\circ}$ & 1,40 & $\begin{array}{l}\text { Sempre (1); } \\
\text { frequentemente } \\
(2) ; \\
\text { frequentemente } \\
\text { (2); sempre (1); } \\
\text { frequentemente } \\
\text { (2). }\end{array}$ & 1,60 & 0,45 & $\begin{array}{l}\text { Flexibilidade de } \\
\text { máquina [4]; } \\
\text { flexibilidade de } \\
\text { volume [1]; } \\
\text { flexibilidade de } \\
\text { expansão [1]; } \\
\text { não há aplicação } \\
\text { de flexibilidade } \\
\text { [1]. }\end{array}$ \\
\hline $\begin{array}{l}\text { Funcionário errar } \\
\text { na mistura para } \\
\text { banho de cabo [2]; } \\
\text { Erro por falta de } \\
\text { compreensão do } \\
\text { processo de banho } \\
\text { pelo funcionário } \\
\text { [1]. }\end{array}$ & 3 & $4^{\circ} ; 2^{\circ} ; 2^{\circ}$ & 2,67 & $\begin{array}{l}\text { Às vezes (3); } \\
\text { frequentemente } \\
(2) ; \\
\text { frequentemente } \\
(2) .\end{array}$ & 2,33 & 2,07 & $\begin{array}{l}\text { Flexibilidade de } \\
\text { mão-de-obra } \\
\text { [3]. }\end{array}$ \\
\hline $\begin{array}{l}\text { Paradas de } \\
\text { produção por } \\
\text { pique de energia } \\
\text { [1]; Perder } \\
\text { produção por } \\
\text { queda de energia } \\
\text { não programada } \\
\text { [1]; Pique de } \\
\text { energia [1]. }\end{array}$ & 3 & $2^{\circ} ; 3^{\circ} ; 1^{\circ}$ & 2,00 & $\begin{array}{l}\text { Às vezes (3); } \\
\text { Raramente (4); } \\
\text { Às vezes (3). }\end{array}$ & 3,33 & 2,22 & $\begin{array}{l}\text { Não há } \\
\text { aplicação de } \\
\text { flexibilidade [3]. }\end{array}$ \\
\hline $\begin{array}{l}\text { Fornecedor } \\
\text { entregar bobina de }\end{array}$ & & & & Às vezes (3); às & & & $\begin{array}{l}\text { Flexibilidade de } \\
\text { máquina [1]; não }\end{array}$ \\
\hline
\end{tabular}




\begin{tabular}{|c|c|c|c|c|c|c|c|}
\hline $\begin{array}{l}\text { baixa qualidade [1]; } \\
\text { bobina de aço fora } \\
\text { das especificações } \\
\text { [1]. }\end{array}$ & 2 & $3^{\circ} ; 1^{\circ}$ & 2,00 & vezes (3). & 3,00 & 3,00 & $\begin{array}{l}\text { há aplicação de } \\
\text { flexibilidade [1] }\end{array}$ \\
\hline $\begin{array}{l}\text { Erros de } \\
\text { planejamento de } \\
\text { vendas [1]; Vendas } \\
\text { determinar } \\
\text { produção de pneu } \\
\text { que não vende [1]. }\end{array}$ & 2 & $3^{\circ} ; 3^{\circ}$ & 3,00 & $\begin{array}{l}\text { Frequentemente } \\
\text { (2); Às vezes (3). }\end{array}$ & 2,50 & 3,75 & $\begin{array}{l}\text { Flexibilidade de } \\
\text { volume [2]; } \\
\text { flexibilidade de } \\
\text { mix [1] }\end{array}$ \\
\hline $\begin{array}{l}\text { Problemas de } \\
\text { comunicação entre } \\
\text { vendas e produção } \\
\text { [1]; Falhas de } \\
\text { produção por erro } \\
\text { de comunicação } \\
\text { [1]. }\end{array}$ & 2 & $5^{\circ} ; 4^{\circ}$ & 4,50 & $\begin{array}{l}\text { Frequentemente } \\
(2) ; \\
\text { frequentemente } \\
(2)\end{array}$ & 2,00 & 4,50 & $\begin{array}{l}\text { Flexibilidade de } \\
\text { produção [1]; } \\
\text { não há aplicação } \\
\text { de flexibilidade } \\
\text { [1]. }\end{array}$ \\
\hline Retrabalho [1]. & 1 & $3^{\circ}$ & 3,00 & As vezes (3) & 3,00 & 9,00 & $\begin{array}{l}\text { Não há } \\
\text { aplicação de } \\
\text { flexibilidade [1]. }\end{array}$ \\
\hline $\begin{array}{l}\text { Problemas } \\
\text { ambientais [1]. }\end{array}$ & 1 & $4^{\circ}$ & 4,00 & Raramente (4) & 4,00 & $\begin{array}{c}16,0 \\
0\end{array}$ & $\begin{array}{l}\text { Flexibilidade de } \\
\text { modificação [1]. }\end{array}$ \\
\hline
\end{tabular}

Fonte: Dados da pesquisa

Como o tema flexibilidade de manufatura é o tópico central dessa pesquisa, ampliou-se o estudo empírico sobre sua compreensão, por meio das Questões 5 e 6 do instrumento de coleta de dados, conforme excerto do mesmo no Quadro 2.

Quadro 2: Excerto do instrumento de coleta de dados aplicado na indústria de pneus

Questão 5. Para cada tipo de flexibilidade, atribua uma nota de 1 a 5, onde 1 indica que a flexibilidade não ajuda em nada na solução de problemas ou não se aplica; 2 indica que ajuda pouco; 3 indica que ajuda razoavelmente; 4 indica que ajuda bastante; e 5 indica que a flexibilidade é imprescindível para a solução de problemas. Para tanto, basta marcar um " $X$ " na pontuação correspondente a cada tipo de flexibilidade.

Questão 6. Se por um lado a empresa necessita investir (gastar dinheiro) para adquirir flexibilidade, por outro lado a flexibilidade de manufatura ajuda a minimizar e resolver alguns tipos de problema nas fábricas. Em tese, quanto mais flexível é uma empresa, 
menos problemas ela terá, porém maiores investimentos serão necessários. A maior dificuldade está nesta dosagem, ou seja, quanto investir e em quais tipos de flexibilidade investir. Desta forma, gostaríamos de saber, na sua opinião, quais tipos de flexibilidade de manufatura você julga indispensável para sua empresa.

Fonte: Dados da pesquisa

As informações obtidas por meio destas duas questões também foram organizadas no Quadro 3, sobre o qual seguem informações sobre seus componentes:

- As colunas '1', '2', '3', '4' e '5', se referem ao grau da avaliação que foi imputado para cada tipo de flexibilidade, onde ' 1 ' significa que a flexibilidade avaliada não ajuda em nada na solução de problemas na manufatura; '2' significa que ajuda pouco; '3' significa que ajuda razoavelmente; '4' significa que ajuda bastante; e '5' significa que a dimensão de flexibilidade de manufatura avaliada é imprescindível. A marcação de cada coluna com uma letra ' $X$ ' indica o julgamento que o respondente fez para cada tipo de flexibilidade;

- A coluna 'média' e 'mediana' apresentam as medidas de centro, oriundas da mensuração estatística das colunas de avaliação anteriormente descritas;

- A coluna 'composição dos tipos de flexibilidade' mostra os tipos de flexibilidade de manufatura que os respondentes, diante da escassez de recursos para investimentos em flexibilidade de manufatura, escolheriam incorporar em seu ambiente de manufatura, formando assim as 'carteiras de flexibilidade', ou seja, as flexibilidades bem mais apropriadas para a solução de problemas na indústria;

- A coluna 'fator flex' calcula a competência de cada tipo de flexibilidade para o tratamento dos problemas que ocorrem na manufatura. Ressalte-se que, quanto maior for o fator flex, mais essencial é aquele tipo de flexibilidade para a solução de problemas na manufatura.

Uma vez explicado como o quadro de Análise de Flexibilidade de Manufatura (Quadro 3) foi construído, parte-se para sua análise e pode-se constatar que:

- As flexibilidades de mão-de-obra, máquina e volume, conforme já descrito anteriormente, são confirmadas aqui como as dimensões mais requeridas pela indústria de 
pneus, sendo aquelas indicadas para comporem uma composição (carteira) de flexibilidades de manufatura para esse segmento industrial;

- No outro extremo, conforme percepção dos respondentes, as flexibilidades de Mix, Modificação, Movimentação, Operação, Produto e Roteamento em nada ajudam na solução de problemas na indústria de pneus. Isso significa que esse tipo de indústria não precisa investir nesses tipos de flexibilidades, uma vez que a natureza de seus problemas demanda outras dimensões;

- $\quad$ A flexibilidade de expansão ajuda, razoavelmente, na solução de problemas;

- $\quad$ A flexibilidade de entrega, timidamente, auxilia na solução de problemas.

Quadro 3: Análise de flexibilidade de manufatura na indústria de pneus

\begin{tabular}{|c|c|c|c|c|c|c|c|c|c|}
\hline \multicolumn{10}{|c|}{ INDÚSTRIA DE PNEUS - ANÁLISE DE FLEXIBILIDADE DE MANUFATURA } \\
\hline \multirow[t]{2}{*}{$\begin{array}{c}\text { Tipos de } \\
\text { flexibilidade }\end{array}$} & \multicolumn{7}{|c|}{$\begin{array}{l}\text { CLASSIFICAÇÃO: Não ajuda (1); Ajuda pouco (2); Ajuda } \\
\text { razoavelmente (3); Ajuda bastante (4); É imprescindível (5) }\end{array}$} & \multirow{2}{*}{$\begin{array}{l}\text { Composição } \\
\text { dos tipos de } \\
\text { flexibilidade }\end{array}$} & \multirow[t]{2}{*}{$\begin{array}{l}\text { Fator } \\
\text { Flex. }\end{array}$} \\
\hline & 1 & 2 & 3 & 4 & 5 & Mediana & Média & & \\
\hline Entrega & & $x$ & $x \times x$ & $x$ & & 3,0 & 3,0 & 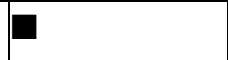 & 3,0 \\
\hline Expansão & & & $x$ & $x x$ & $x x$ & 4,0 & 4,2 & $\mathbf{a}$ & 8,4 \\
\hline Máquina & & & & $x x$ & $x \times x$ & 5,0 & 4,6 & an & 18,4 \\
\hline Mão-de-obra & & & & $x x$ & $x \times x$ & 5,0 & 4,6 & | & 23,0 \\
\hline Mix & & & $x \times x x$ & $x$ & & 3,0 & 3,2 & & 0,0 \\
\hline Modificação & $x$ & $x$ & $x \times x$ & & & 3,0 & 2,4 & & 0,0 \\
\hline Movimentação & $x$ & $x$ & $x \times x$ & & & 3,0 & 2,4 & & 0,0 \\
\hline Operação & $x$ & $x$ & $x \times x$ & & & 3,0 & 2,4 & & 0,0 \\
\hline Produção & & & $x \times x \times$ & & $x$ & 3,0 & 3,4 & $\square$ & 3,4 \\
\hline Produto & $x$ & $x x$ & $x x$ & & & 2,0 & 2,2 & & 0,0 \\
\hline Roteamento & $x$ & $x$ & $x \times x$ & & & 3,0 & 2,4 & & 0,0 \\
\hline Volume & & & & $x \times x$ & $x x$ & 4,0 & 4,4 & an & 17,6 \\
\hline
\end{tabular}

Fonte: Dados da pesquisa 


\section{Conclusões}

Durante o desenvolvimento do referencial teórico, verificou-se que há, de fato, poucos estudos que abordam os riscos operacionais ligados à manufatura, sendo esta uma lacuna que se pretendeu preencher com a presente pesquisa. Dos vários trabalhos consultados (a grande maioria deles não referenciada, por limitações de espaço), aproximadamente metade abordava riscos financeiros, $20 \%$ abordavam riscos oriundos de acidente do trabalho, $20 \%$ abordavam riscos ambientais e, somente $10 \%$ abordavam outros tipos de riscos, incluindo-se, os riscos operacionais. Assim, fica evidenciada a constatação de alguns autores sobre o tema, que afirmam haver muitas pesquisas de riscos na área financeira, porém pouca ou quase nenhuma atenção tem sido dada aos riscos na manufatura (OLIVEIRA, 2009; BARALDI, 2005; FAMA et al., 2002).

Constatou-se, também, na presente pesquisa, que a flexibilidade de manufatura atua como uma fonte de vantagem competitiva para administrar a operação sob condições de mudanças, variedade, riscos e incertezas, permitindo que o processo produtivo continue o seu trabalho, a um baixo custo e com uma resposta mais rápida.

No que se refere ao estudo empírico, observou-se que os problemas mais severos que ocorrem na manufatura da indústria de pneus estão relacionados, por ordem de prioridade, com indisponibilidade de equipamentos, falha humana e fornecedores, confirmando-se, assim, a primeira conjectura da presente pesquisa (vide Figura 1). Este pré-conhecimento é desejado, uma vez que o entendimento dos problemas que mais requerem controle gerencial auxilia no processo de sistematização da monitoração e contramedidas dos riscos.

Por outro lado, analisando-se a frequência que os problemas ocorrem na manufatura de uma indústria de pneus, refuta-se, em parte, a segunda conjectura, uma vez que os problemas mais frequentes estão relacionados com indisponibilidade de máquinas, falhas humanas e erros de comunicação (vide sexta coluna do Quadro 1)

Quanto às flexibilidades de manufatura para a solução dos problemas apontados na empresa pesquisada, verificam-se as de máquina e mão-de-obra como as mais requeridas, corroborando-se, dessa forma, a terceira conjectura desse estudo (vide Quadro 3). Diante disto, caso alguma outra empresa deste segmento empresarial não disponha de tempo, pessoal capacitado, capital, entre outros fatores para desenvolver uma pesquisa em seu próprio ambiente de manufatura, os resultados aqui apresentados fornecem boas indicações de quais flexibilidades poderiam ser utilizadas em seus processos produtivos. Todavia, recomenda-se o aprofundamento em estudos de flexibilidade em mais empresas desse mesmo ramo, com maior grau de propriedade, definir quais tipos de flexibilidade adotar e para quais tipos de problemas.

Por fim, ao analisar quais tipos de flexibilidade os especialistas entrevistados selecionariam para compor uma "carteira de flexibilidade", observa-se a escolha dos seguintes tipos: flexibilidade de mão-de-obra, flexibilidade de máquina e flexibilidade de volume. Apesar 
de não haver dúvidas quanto às escolhas dos entrevistados, esse resultado não é conclusivo para aceitar a quarta conjectura, motivo pelo qual recomenda-se que esse assunto seja aprofundado em pesquisas futuras.

Por fim, analisando-se os objetivos da pesquisa, julga-se que o estudo desenvolvido para a empresa em questão atingiu seu propósito, uma vez que foram levantados os principais problemas que ocorrem em sua manufatura, bem como as dimensões de flexibilidades requeridas para a solução desses problemas.

Ressalta-se que a quarta conjectura não foi avaliada nesse estudo.

\section{Referências}

BARALDI, P. Gerenciamento de Riscos Empresariais. Rio de Janeiro: Campus, 2005.

BAYKASOGLU, A. \& OZBAKIR, L. Analysing the effect of flexibility on manufacturing systems performance. Journal of Manufacturing Technology Management, v. 19, n. 2, 2008, pp. 172-193.

BEACH，R.; MUHLEMANN， A.P.; PRICE， D.H.R.; PATERSON, A. \& SHARP, J.A. A review of manufacturing Flexibility. European Journal of Operational Research, v. 122, 2000, pp. 41-57.

BENGTSSON, J. \& OLHAGER, J., The impact of the product mix on the value of flexibility. The international journal of management science, v. 30 n. 4, 2002, pp. 265-273.

BOLWIJN, P. T. \& KUMP, T. Manufacturing in the 1990s - productivity, flexibility and innovation. Long Range Planning, v. 23, n. 4, 1990 pp. 44-57.

BOYLE, T. A. Towards best management practices for implementing manufacturing flexibility. Journal of Manufacturing Technology Management, v. 17, n. 1, 2006 pp. 6-21.

BROWNE, J.; DUBOIS, D.; RATHMILL, K.; SETHI, S. $P$. \& STECKE, K. Types of flexibilities and classification of flexible manufacturing systems. Working Paper, n. 367. Graduate
School of Business Administration, University of Michigan, 1984.

CHANG, S.; LIN, R.; CHANG, F. \& CHEN, R. Achieving manufacturing flexibility through entrepreneurial orientation. Industrial Management \& Data Systems, v. 107, n. 7, 2007 pp. 997-1017.

CHOE， P.; TEW， J. D.; TONG， S. Effect of cognitive automation in a material handling system on manufacturing flexibility. International Journal of Production Economics, Vol 170, 2015, pp. 891-899.

CORRÊA, H. L. Flexibilidade estratégica na manufatura: incertezas e variabilidade de saída. Revista de Administração da USP, v. 29, n. 1, 1994

D'SOUZA, D. E. \& WILLIAMS, F. P. Toward a taxonomy of manufacturing flexibility dimensions. Journal of Operations Management, v. 18, 2000, pp. 577-593.

DE OLIVEIRA, U.R.; MARINS, F. A. S.; ROCHA, H. M. SALOMON, V. A. P. The ISO 31000 standard in supply chain risk management. Journal of Cleaner Production. Vol. 151, 10, 2017. pp. 616633.

DE TONI, A. \& TONCHIA, S. Manufacturing flexibility: a literature review. International 
Journal of Production Research. V. 36, n. 6, 1998, pp. 1587-1617.

DE TREVILLE, S; BENDAHAN， S. \& VANDERHAEGHE, A. Manufacturing flexibility and performance: bridging the gap between theory and practice. International Journal of Flexibility Manufacturing Systems, v. 19, 2007, pp. 334-357.

FAMÁ, R.; CARDOSO, R. L. \& MENDONÇA, O. Riscos Financeiros e não Financeiros: Uma Proposta de Modelo para Finanças. Cadernos da FACECA v. 11 n. 1 jan/jun 2002, pp. 33-50.

FERNANDES, R.; GOUVEIA， J. B. PINHO, C. Product mix strategy and manufacturing flexibility. Journal of Manufacturing Systems. Vol. 31, 2012, pp. 301- 311.

FRYER, J. S. Labor flexibility in multiechelon dual-constrained job shops. Management Science, v. 20 n. 7, 1974 pp. 1073-1080.

GERWIN, D. Manufacturing flexibility: a strategic perspective. Management Science; v. 39, n. 4, 1993, pp. 395-410

GERWIN, D. An agenda for research on the flexibility of Manufacturing processes. International Journal of Operations \& Production Management, v. 25, n. 12, 2005 pp. 1171-1182.

GUPTA， Y. P. \& GOYAL, S. Flexibility of manufacturing systems: concept and measurements. European Journal of Operational Research, v. 40, 1989, pp. 119-135.

GUPTA, Y. P. \& SOMERS, T. M. The measurement of manufacturing flexibility. European Journal of Operational Research, v. 60, 1992, pp. 166-192.

GUSTAVSSON, S. Flexibility and productivity in complex production processes.
International Journal of Production Research, v. 22, n. 5, 1984, pp. 801-808.

HE, Y.; LAI, K. K.; SUN, H.; CHEN, Y. The impact of supplier integration on customer integration and new product performance: The mediating role of manufacturing flexibility under trust theory. International Journal of Production Economics, Vol. 147, 2014, pp. 260-270.

JAVIER， T.; GUTIERREZ-GUTIERREZ， L.; RUIZMORENO, $A$. The relationship between exploration and exploitation strategies, manufacturing flexibility and organizational learning: An empirical comparison between Non-ISO and ISO certified firms. European Journal of Operational Research, Vol. 232, 2014, pp. 72-86.

KARA, S. \& KAYIS, B. Manufacturing flexibility and variability: an overview. Journal of Manufacturing Technology Management, v.15, n. 6, 2004, pp. 466-478.

KAYIS, B. \& KARA, S. The supplier and customer contribution to manufacturing flexibility: Australian manufacturing industry's perspective. Journal of Manufacturing Technology Management, v. 16, n. 7, 2005 pp. 733-752.

KOSTE, L. L.; MALHOTRA, M. K. A theoretical framework for analyzing the dimensions of manufacturing flexibility. Journal of Operations Management, v.18, 1999, pp. 75-93.

MARCONI, M. A. \& LAKATOS, M.E. Metodologia Científica. $4^{a}$ edição. São Paulo: Atlas, 2004.

MILLER, D. \& SHAMSIE, J. Strategic responses to three kinds of uncertainty: product line simplicity at the Hollywood film studios. 
Journal of Management v. 25, n. 1, 1999, pp. 97116.

MISHRA, R. A comparative evaluation of manufacturing flexibility adoption in SMEs and large firms in India. Journal of Manufacturing Technology Management, Vol. 27 No. 5, 2016, pp. 730-762.

MISHRA, R.; PUNDIR, A. K.; GANAPATHY, L. Conceptualizing sources, key concerns and critical factors for manufacturing flexibility adoption: An exploratory study in Indian manufacturing firms. Journal of Manufacturing Technology Management, Vol. 27 No. 3, 2016, pp. 379-407.

NARAIN, R.; YADAV, R.C; SARKIS, J. \& CORDEIRO, J. J. The strategic implications of flexibility in manufacturing systems. International Journal of Agile Management Systems, v. 2, n. 3, 2000, pp. 202-213.

NARASIMHAN, R.; TALLURI, S. \& DAS, A. Exploring flexibility and execution competencies of manufacturing firms. Journal of Operations Management, v. 22, 2004, pp. 91106.

NILSSON, C. H. \& NORDAHL, H. Making manufacturing flexibility operational - part 1: a framework. Integrated Manufacturing Systems, v. 6, n 1, 1995, pp. 5-11.

NORDAHL, H.; NILSSON, C. Managers' perceptions of flexibility in manufacturing: a study in the Swedish engineering industry. Integrated Manufacturing Systems, v.7, n.4, 1996, pp. 22-33.

$O K E$, A. A framework for analyzing manufacturing flexibility. International Journal of Operations \& Production Management, Vol. 25, n. 10, 2005 pp. 973-996.
$\mathrm{OKE}, \mathrm{A}$. Linking manufacturing flexibility to innovation performance in manufacturing plants. International Journal of Production Economics, Vol. 143, 2013, pp. 242-247.

OLHAGER J. \& WEST B. M. The house of flexibility: using the QFD approach to deploy manufacturing flexibility. International Journal of Operations and Production Management, v. 22, n 1, 2002, pp. 50-79.

OLHAGER, J. Manufacturing flexibility and profitability. International Journal of Production Economics v. 30-31, 1993, pp. 67-78.

OLIVEIRA, U. R. Gerenciamento de riscos operacionais na indústria por meio da seleção de diferentes tipos de flexibilidade de manufatura. 2009. 246 f. Tese (Doutorado em Engenharia Mecânica) - Faculdade de Engenharia do Campus de Guaratinguetá, Universidade Estadual Paulista, Guaratinguetá, 2009.

PADOVEZE, C. L. \& BERTOLUCCI, R. G. Proposta de um Modelo para o Gerenciamento do Risco Corporativo. Anais XXV Encontro Nacional de Engenharia de Produção, Porto Alegre, 2005.

PAGELL, M. \& KRAUSE, D. R. A multiplemethod study of environmental uncertainty and manufacturing flexibility. Journal of Operations Management, v. 17, 1999, pp. 307325.

PAGELL, M. \& KRAUSE, D. R. Re-exploring the relationship between flexibility and the external environment. Journal of Operations Management, v. 21, 2004, pp. 629-649.

PANHOCA, L. Administração do risco de propostas e estudos de viabilidade na indústria aeronáutica brasileira: uma abordagem de controladoria. Tese de Doutorado da Faculdade de Economia, 
Administração e Contabilidade da Universidade de São Paulo, São Paulo, 2000.

PATEL, P. Role of manufacturing flexibility in managing duality of formalization

and environmental uncertainty in emerging firms. Journal of Operations Management, Vol. 29, 2011, pp. 143-162

PATEL, P. C., TERJESEN, S.; LI, D. Enhancing effects of manufacturing flexibility through operational absorptive capacity and operational ambidexterity. Journal of Operations Management, Vol. 30, 2012, pp. 201-220.

PURWANTO, U. S.; KAMARUDDIN, S. MOHAMAD, $N$. The Role of Industrial Clustering and Manufacturing Flexibility in Achieving High Innovation Capability and Operational Performance in Indonesian Manufacturing SMEs. Industrial Engineering \& Management Systems, Vol 14, No 3, 2015, pp.236-247.

SAVARIS, C. E. Modelo para identificação e avaliação do impacto dos recursos tecnológicos, organizacionais e de suprimentos na flexibilidade da manufatura. Dissertação Mestrado. Programa de PósGraduação em Engenharia de Produção, UFSC, Florianópolis, 2003.

SAWHNEY, R. Interplay between uncertainty and flexibility across the value-chain: Towards a transformation model of manufacturing flexibility. Journal of Operations Management, v. 24, 2005, pp. 476493.

SCALA, J; PURDY, L \& SAFAYENI, F. Application of cybernetics to manufacturing flexibility: a systems perspective. Journal of Manufacturing
Technology Management, v. 17, n. 1, 2006, pp. 22-41.

SERRÃO, R. O. B. Um estudo sobre a flexibilidade de manufatura e sua percepção e efetivação em micro e pequenas empresas. Dissertação de Mestrado. Departamento de Engenharia Industrial. Pontifícia Universidade Católica do Rio de Janeiro, 2001.

SLACK, N. Vantagem competitiva em manufatura: atingindo a competitividade nas operações industriais. São Paulo: Atlas, 1993.

SLACK, N. Flexibility as a manufacturing objective. International Journal of Operations and Production Management 33, 1983, pp. 413.

SLACK, N. The flexibility of manufacturing systems. International Journal of Operations \& Production Management, v. 25, n. 12, 2005 pp. 1190-1200.

SUAREZ, F. F.; CUSUMANO, M. A. \& FINE, C. H. An Empirical Study of Flexibility in Manufacturing. Sloan Management Review, v. 37, n. 1, 1995, pp. 25-32.

SWAMIDASS, P. M. \& NEWELL, W. T. Manufacturing strategy, environmental uncertainty and performance: a path analytic model. Management Science, v. 33, n. 4, 1987, pp. 509-524.

UPTON, D. M. Flexibility as process mobility: The management of plant capabilities for quick response manufacturing. Journal of Operations Management, v. 12, 1995, pp. 205224.

UPTON, D.M. Process range in manufacturing: an empirical study of flexibility. Management Science, v. 43, 1997, pp. 1079-1092.

UPTON, D. M. The management of manufacturing flexibility. California 
Management Review, v. 36, n. 2, 1994, pp. 7289.

URTASUN-ALONSO, A.; LARRAZA-KINTANA, M.; GARCÍA-OLAVERRI， C.; HUERTA-ARRIBAS， E. Manufacturing flexibility and advanced human resource management practices, Production Planning \& Control, 2014, Vol. 25, No 4, pp.303-317.

VOKURKA, R. J. \& O'LEARY-KELLY, S. W. A review of empirical research on manufacturing flexibility. Journal of
Operations Management, v. 18, 2000, pp.485501.

WAHAB, M. I. M.; WU, D. \& LEE, C. A generic approach to measuring the machine flexibility of manufacturing systems. European Journal of Operational Research, v. 186, 2008, pp. 137-149.

WATTS, C., HAHN, C. \& SOHN, B. Manufacturing flexibility: concept and measurement. Operations Management Review. v. 9 n. 4, 1993, pp. 33-44.

Recebido em: 15/08/2019

Aceito em: 14/10/2019

Endereço para correspondência:

Ualison Rebula de Oliveira

ualison.oliveira@gmail.com

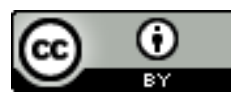

Esta obra está licenciada sob uma Licença Creative Commons Attribution 3.0 